\title{
A Polarization Independent Hybrid Coupler for Silicon on Insulator Waveguides
}

\author{
M. Z. Alam, J. S. Aitchison and M. Mojahedi \\ Department of Electrical and Computer Engineering, \\ University of Toronto, Toronto, Ontario, Canada, MSS $3 G 4$ \\ malam@waves.utoronto.ca
}

\begin{abstract}
The polarization dependence of a hybrid waveguide consisting of a high index medium adjacent to a metal with a low index spacer is described. We present the design of a polarization independent hybrid waveguide coupler.

(C)2010 Optical Society of America

OCIS codes: (310.6628) Subwavelength structures, nanostructures; (130.3120) Integrated optics
\end{abstract}

\section{Introduction}

Silicon on insulator (SOI) photonics has attracted much interest in recent years due to the potential for integration with silicon CMOS. One of the major limitations of SOI devices is their high polarization dependency. In most optical fiber system polarization is a randomly changing quantity therefore to ensure proper functioning and integration of fibers and SOI devices, care must be taken. One possible solution is to use polarization diversity scheme where the two polarizations are separated and processed independently or one polarization is converted to the other. This requires the use of additional components like polarization beam splitters and polarization rotators which increases system size and complexity. Another approach is to make the devices polarization independent. Although realization of polarization independent straight SOI waveguides is relatively straight forward [1], doing the same for SOI devices is more challenging. For example, directional coupler - a very important component of many integrated optic circuits is inherently polarization dependent. To date there have been very few reports on polarization independent directional couplers [2]. Here we present the design of a polarization independent directional coupler using the recently proposed hybrid waveguide [3-5].

\section{Principle of operation}

Figure 1 (a) shows the hybrid waveguide which is the building block of the directional coupler. It consists of a silicon slab of dimensions $w \times d$ separated from a silver top layer of dimension $w \times t$ by a silica spacer layer of dimension $w \times h$. The surrounding medium is air. Figure 1(b) and 1(c) shows the guided power density profiles for the TM and TE modes at $1550 \mathrm{~nm}$ wavelength calculated by commercial finite element code Comsol Multiphysics. The properties for silica, silver, and silicon are taken from [6]. For the TM mode most power is confined in the low index spacer layer but for the TE mode power is mostly confined in the silicon slab.

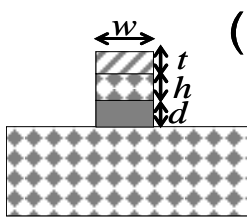

(a)
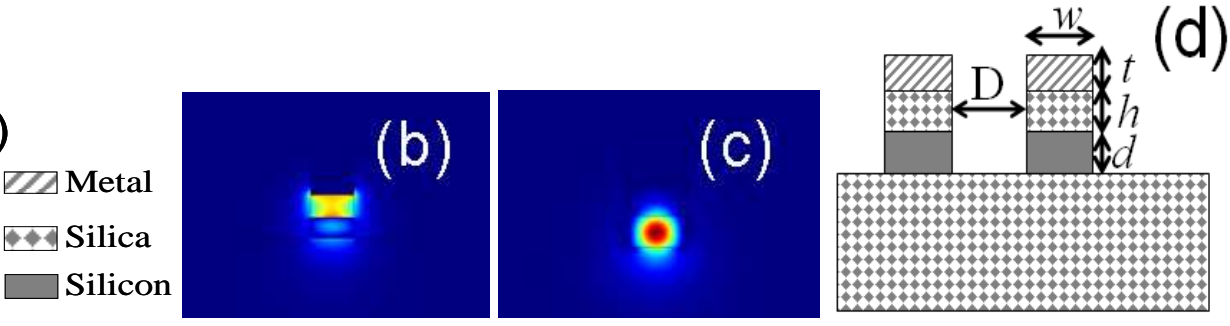

Fig. 1. (a) Hybrid waveguide. (b) Guided power density for TM mode (b) Guided power density for TE mode. Waveguide dimensions are $w=350$ $\mathrm{nm}, t=250 \mathrm{~nm}, h=190 \mathrm{~nm}, d=170 \mathrm{~nm}(\mathrm{~d})$ Polarization independent directional coupler

When two identical hybrid guides are separated by a small distance $D$, as shown in Fig. 1(d), the modes guided by the two waveguides will couple and power transfer between the waveguides will take place. Coupling length i.e. the length required for complete power transfer from one guide to the other is $L=\pi /\left(\beta_{\text {even }}-\beta_{\text {odd }}\right)$. Here $\beta_{\text {even }}$ and $\beta_{\text {odd }}$ are the effective indices of the even and odd modes of the coupled waveguides of Fig. 1 (d). The coupling length of the modes depends on their confinement. Lack of confinement results in increased coupling and hence reduced coupling length. Since the fields for the two polarizations are confined in two different layers, changing the waveguide dimensions affects the confinement of the two modes in different manners. By choosing the dimensions properly it 


\section{FThC3.pdf}

is possible to have equal level of coupling and hence equal coupling lengths for both polarizations. This is the working principle of the proposed polarization-independent coupler.

\section{Analysis and results}

The important design parameters here are waveguide width $w$, silicon height $d$, spacer height $h$ and waveguide spacing $D$. Figure 2 shows the effects of variation of $h$ and $d$ on coupling length for TE and TM modes. With increasing $d$, both modes are more tightly confined and coupling length increases. The increase is much faster for TE mode than for TM mode. Spacer height $h$ affects the two modes in two different manners. In case of TE mode, for a small $h$ the field is pushed out of the core and coupling length is reduced. The TM mode on the other hand becomes more tightly confined in the spacer layer for small $h$ which increases its coupling length. For $d=175 \mathrm{~nm}$, $h=175 \mathrm{~nm}, w=350 \mathrm{~nm}$, coupling lengths become same for both modes and the device becomes polarization independent. The coupling length in this case is approximately $20 \mu \mathrm{m}$.

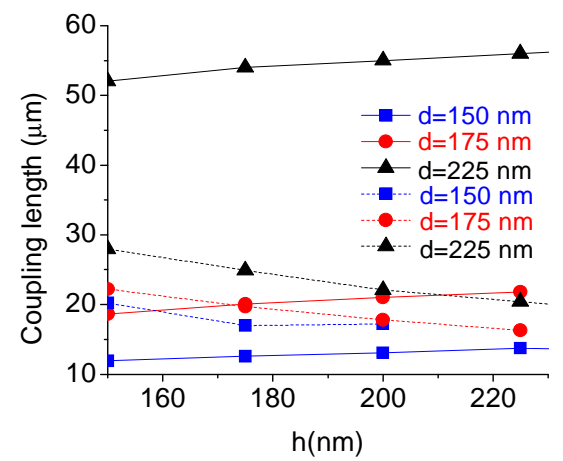

Fig.2 Variations of coupling length with h for various fixed values of d. Metal height is $200 \mathrm{~nm}$ and waveguide spacing is $500 \mathrm{~nm}$. Solid lines are for TE mode and dashed lines are for TM modes.

Fig. 3 shows the variation of coupling length as a function of waveguide spacing $D$. The device length can be made less than $10 \mu \mathrm{m}$ by suitable choice $D$.

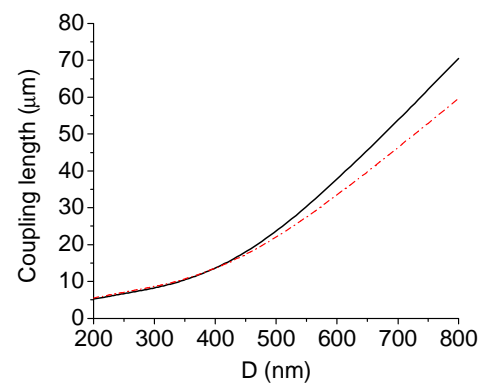

Fig. 3. Variation of coupling length with waveguide spacing $D$. Waveguide dimensions are $h=175 \mathrm{~nm}, d=175 \mathrm{~nm}, t=200 \mathrm{~nm}, w=350 \mathrm{~nm}$. Solid line is for TE mode and dashed line is for TM mode

In conclusion we have proposed a polarization independent directional coupler which is very compact and compatible with SOI technology.

\section{References}

[1] S. T. Lim, C. E. Png, E. A. Ong, "Single mode polarization independent submicron silicon waveguide based on geometrical adjustments", Optics Express, 15(18): 11061-11072, 2007.

[2]T. Fujisawa, M. Koshiab, "Polarization-independent optical directional coupler based on slot waveguides", Optics Letters, 31(1): 56-58, 2006.

[3] M Z. Alam, J Meier, J S. Aitchison, M Mojahedi, "Super mode propagation in low index medium," CLEO/QELS 2007, http://www.opticsinfobase.org/abstract.cfm?uri=CLEO-2007-JThD112.

[4] R. F. Oulton, V. J. Sorger, D. A. Genov, D. F. P. Pile, X. Zhang, "A hybrid plasmonic waveguide for subwavelength confinement and long range propagation," Nature Photonics, 2: 496-500, (2008).

[5] D. Dai, S. He, "A silicon-based hybrid plasmonic waveguide with a metal cap for a nano-scale light confinement", Optics Express, 17(19): 16646-16652, 2009.

[6] E .D. Palik, Handbook of optical constants of solids, (Academic Press, Inc. 1985). 